Article

\title{
Area-Specific Cell Stimulation via Surface-Mediated Gene Transfer Using Apatite-Based Composite Layers
}

\author{
Yushin Yazaki ${ }^{1,2}$, Ayako Oyane ${ }^{2, *}$, Yu Sogo $^{3}$, Atsuo Ito ${ }^{3}$, Atsushi Yamazaki ${ }^{1}$ \\ and Hideo Tsurushima ${ }^{2,4}$
}

1 Department of Resources and Environmental Engineering, Waseda University, 3-4-1 Okubo, Shinjuku, Tokyo 169-8555, Japan; E-Mails: yushin_0822@yahoo.co.jp (Y.Y.); ya81349@waseda.jp (A.Y.)

2 Nanomaterials Research Institute, National Institute of Advanced Industrial Science and Technology, Central 4, 1-1-1 Higashi, Tsukuba, Ibaraki 305-8562, Japan

3 Health Research Institute, National Institute of Advanced Industrial Science and Technology, Central 6, 1-1-1 Higashi, Tsukuba, Ibaraki 305-8566, Japan;

E-Mails: yu-sogou@aist.go.jp (Y.S.); atsuo-ito@aist.go.jp (A.I.)

4 Department of Neurosurgery, Faculty of Medicine, University of Tsukuba, 1-1-1 Tennoudai, Tsukuba, Ibaraki 305-8575, Japan; E-Mail: hideo-tsurushima@md.tsukuba.ac.jp

* Author to whom correspondence should be addressed; E-Mail: a-oyane@aist.go.jp; Tel.: +81-29-861-4693; Fax: +81-29-861-3005.

Academic Editor: Mohamed N. Rahaman

Received: 18 November 2014 / Accepted: 30 March 2015 / Published: 14 April 2015

\begin{abstract}
Surface-mediated gene transfer systems using biocompatible calcium phosphate (CaP)-based composite layers have attracted attention as a tool for controlling cell behaviors. In the present study we aimed to demonstrate the potential of CaP-based composite layers to mediate area-specific dual gene transfer and to stimulate cells on an area-by-area basis in the same well. For this purpose we prepared two pairs of DNA-fibronectin-apatite composite (DF-Ap) layers using a pair of reporter genes and pair of differentiation factor genes. The results of the area-specific dual gene transfer successfully demonstrated that the cells cultured on a pair of DF-Ap layers that were adjacently placed in the same well showed specific gene expression patterns depending on the gene that was immobilized in theunderlying layer. Moreover, preliminary real-time PCR results indicated that multipotential $\mathrm{C} 3 \mathrm{H} 10 \mathrm{~T} 1 / 2$ cells may have a potential to change into different types of cells depending on the differentiation factor gene that was immobilized in the underlying layer,
\end{abstract}


even in the same well. Because DF-Ap layers have a potential to mediate area-specific cell stimulation on their surfaces, they could be useful in tissue engineering applications.

Keywords: dual gene transfer; hydroxyapatite; reverse transfection; calcium phosphate; scaffold

\section{Introduction}

Safe, efficient, and area-specific gene transfer systems for controlling cell behaviors, including proliferation and differentiation, are useful tools for tissue engineering. Conventional nonviral gene transfer systems are generally mediated by particulate complexes of DNA and transfection reagents, such as lipids [1-3], cationic polymers [4-7], and calcium phosphates (CaPs) [8-13]. These particle-mediated systems are usually weak in area-specific gene transfer and are incapable of stimulating cells at a specific location. This is because particulate DNA complexes easily disperse in a culture medium or in body fluids.

Since the late 1990s, surface-mediated gene transfer systems (also called reverse transfection) that are capable of area-specific gene transfer have been developed [14-32]. In these systems, the cells are seeded onto substrates with immobilized DNA and, in many cases, with transfection reagents as well. The immobilized DNA is released from the substrate and then taken up by the cells that have adhered to the substrate. Recently, other approaches that use physical stimulations, including electric pulses [33-35], ultrasound [36-38], and atmospheric plasmas [39-41], have also been proposed for achieving area-specific gene transfer.

Among such surface-mediated gene transfer systems, we have focused on a system that uses CaP-based composite layers [18-27] toward in vitro and in vivo tissue engineering applications. $\mathrm{CaP}$-based composite layers consist of a matrix of $\mathrm{CaP}$ with immobilized DNA and can be coated onto a variety of scaffold materials [25]. Owing to the good biocompatibility of CaP, these composite layers support cell viability on their surfaces with minimal toxicity [18]. Furthermore, these composite layers can be designed to exhibit increased gene transfer efficiency by coimmobilization of biofunctional molecules such as cell adhesion molecules (laminin [19,20], fibronectin [21,22]), and lipids [23,24,26,27] within the composite layer. We have demonstrated that CaP-based composite layers have the potential to accelerate not only in vitro cell differentiation [20,42,43], but also in vivo bone tissue regeneration [42] on the layers. Our preliminary studies showed that a DNA-fibronectin-apatite composite layer (DF-Ap layer) potentially allow area-specific gene transfer on their surfaces using a simple assay system based on a luciferase reporter gene [21]. Area-specific gene transfer was suggested by the observation that the cells cultured on the composite layer shows significantly higher luciferase activity than the cells cultured on the well around the composite layer in the same well [21].

In the present study, we aimed to demonstrate the potential of CaP-based composite layers to mediate area-specific dual gene transfer and cell stimulation in the same well. For this purpose, we designed two experimental systems using two pairs of DF-Ap layers: a pair of layers immobilizing reporter genes for area-specific dual gene transfer study and pair of layers immobilizing differentiation factor genes for area-specific cell stimulation (Table 1). 
Table 1. List of the genes, plasmid DNAs, sample names, and cell lines used in this study.

\begin{tabular}{|c|c|c|c|c|}
\hline Type of Gene & Gene & Plasmid DNA & Sample Name & Transfected Cell \\
\hline \multirow{2}{*}{ Reporter gene } & Firefly luciferase (FL) & pGL3 $^{(1)}$ & DF-FL & \multirow{2}{*}{ CHO-K1 cell } \\
\hline & Renilla luciferase $(R L)$ & pRL-TK ${ }^{(2)}$ & DF-RL & \\
\hline \multirow{2}{*}{$\begin{array}{l}\text { Differentiation } \\
\text { factor gene }\end{array}$} & $\begin{array}{l}\text { Vascular endothelial } \\
\text { growth factor (VEGF) }\end{array}$ & pCI-VEGF $^{(3)}$ & DF-V & \multirow{2}{*}{$\begin{array}{l}\text { C3H10T1/2 } \\
\text { embryonic cell }\end{array}$} \\
\hline & $\begin{array}{c}\text { Bone morphogenetic } \\
\text { protein-2 }(B M P-2)\end{array}$ & $\mathrm{pCI}-\mathrm{BMP}^{(4)}$ & DF-B & \\
\hline
\end{tabular}

(1) pGL3: pGL3-Control vector including the cDNA of firefly luciferase $(F L)$; ${ }^{(2)}$ pRL-TK: pRL-TK vector including the cDNA of Renilla luciferase $(R L) ;{ }^{(3)}$ pCI-VEGF: pCI-neo vector including the cDNA of human vascular endothelial growth factor (VEGF); ${ }^{(4)} \mathrm{pCI}$-BMP: pCI-neo vector including the cDNA of human bone morphogenetic protein-2 (BMP-2).

First, we prepared two types of DF-Ap layers, each of which had a different reporter gene: the cDNA of firefly luciferase $(F L)$ or of Renilla luciferase $(R L)$. Each DF-Ap layer was fabricated on a polystyrene substrate via a precursor-assisted biomimetic process using a simplified alternate dipping treatment [21,25]. The resulting substrates (termed Samples DF-FL and DF-RL, as listed in Table 1) with the DF-Ap layers were adjacently placed and used as scaffolds for culturing Chinese hamster ovary-K1 (CHO-K1) cells to allow area-specific dual gene transfer in the same well (Figure 1). We employed the CHO-K1 cell line, because it is well-characterized, easy to culture and transfect, and has been used in our previous studies [21,22,26,27]. The FL and RL activities of the cells cultured on each DF-Ap layer were evaluated by a dual luciferase assay system.

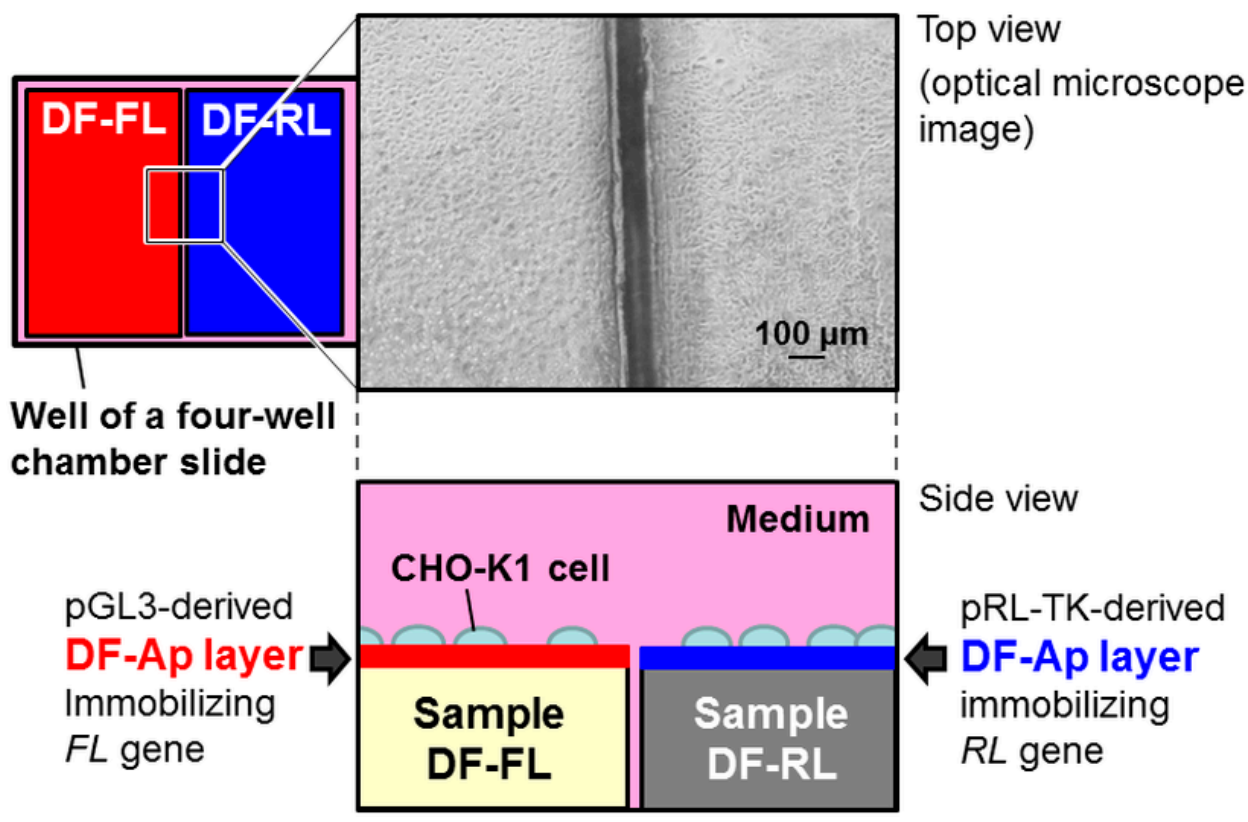

Figure 1. Schematic depiction of the sample setting used in the dual reporter gene transfer study.

Second, we used the precursor-assisted biomimetic process to prepare another set of two DF-Ap layers, each of which had a different differentiation factor gene: the cDNA of the vascular endothelial growth factor (VEGF) [44] or of the bone morphogenetic protein-2 (BMP-2) [45]. The resulting 
substrates (termed Samples DF-V and DF-B, as listed in Table 1) with the DF-Ap layers were then applied to preliminary dual gene transfer studies sing an experimental system that was similar to that illustrated in Figure 1 and multipotential C3H10T1/2 cells. The C3H10T1/2 cells are VEGF- and BMP-2-responsive mouse embryonic cells. VEGF and BMP-2 are vascular endothelial and osteogenic differentiation factors, respectively. The C3H10T1/2 cells cultured on each DF-Ap layer were assayed for $V E G F, B M P-2$, and differentiation marker gene expression by real-time PCR.

\section{Results}

\subsection{Surface Structures of Samples}

The formation of DF-Ap layers on the surfaces of Samples DF-FL and DF-RL was confirmed by scanning electron microscopy (SEM), thin-film X-ray diffractometry (TF-XRD), and chemical analyses by inductively coupled plasma atomic emission spectrometry (ICP) and ultraviolet-visible spectrophotometry (UV-Vis). Uniform layers with a microscale cavernous and lumpy structure, as shown in the SEM images in Figure 2, were observed over the entire surface of both samples. No noticeable differences in surface morphologies were observed under SEM between these two layers. These layers were composed of low-crystalline apatite, as revealed by their TF-XRD patterns (Figure 3). The amount of apatite was comparable in Samples DF-FL and DF-RL because there was no significant difference in calcium and phosphorus contents on their surfaces (Figure 4a). The two apatite layers on Samples DF-FL and DF-RL immobilized plasmid DNA and fibronectin to a similar extent, according to the UV-Vis results (Figure 4b). These results indicate that the DF-Ap layers formed on Samples DF-FL and DF-RL were comparable in physicochemical properties, although the immobilized plasmid DNA was different (pGL3 in Sample DF-FL and pRL-TK in Sample DF-RL).
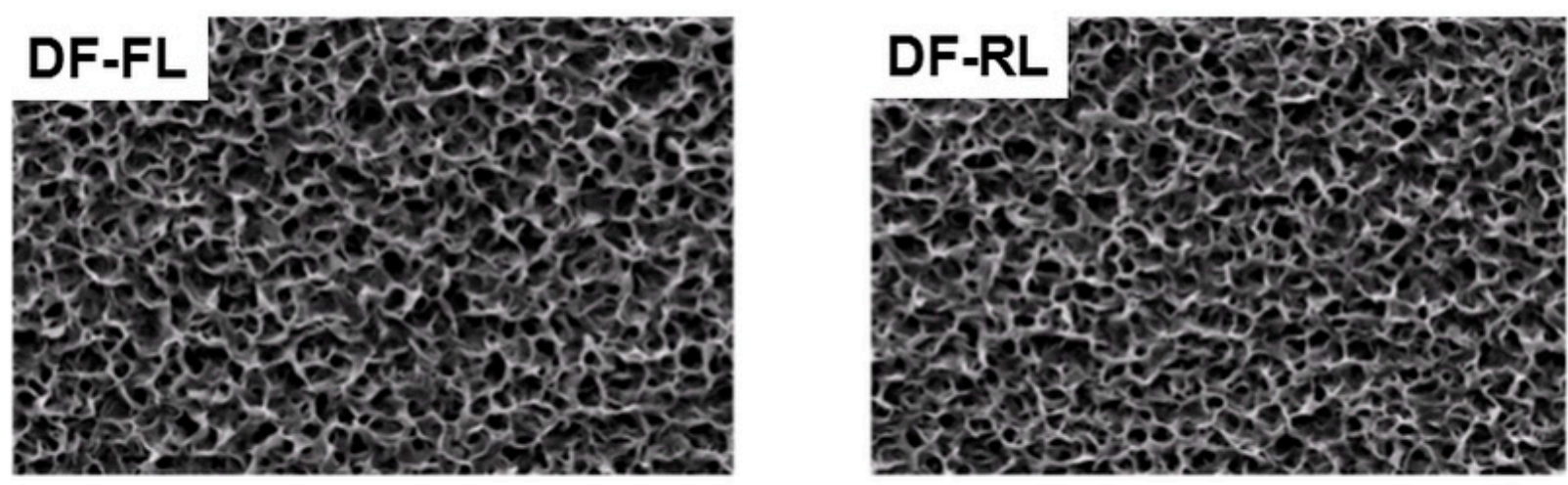

\section{$5 \mu \mathrm{m}$}

Figure 2. Scanning electron microscopy (SEM) images of the surfaces of the DNA-fibronectin-apatite composite layers (DF-Ap layers) immobilizing firefly luciferase $(F L)$ gene (Sample DF-FL) and Renilla luciferase $(R L)$ gene (Sample DF-RL). 


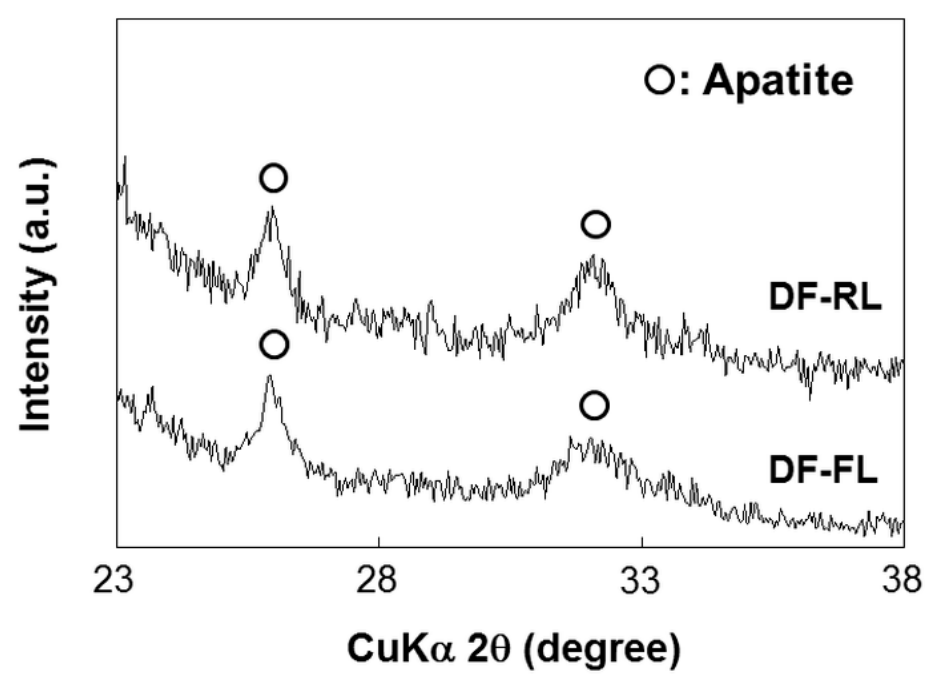

Figure 3. Thin-film X-ray diffraction (TF-XRD) patterns of the surfaces of the DNA-fibronectin-apatite composite layers (DF-Ap layers) immobilizing firefly luciferase $(F L)$ gene (Sample DF-FL) and Renilla luciferase ( $R L)$ gene (Sample DF-RL).

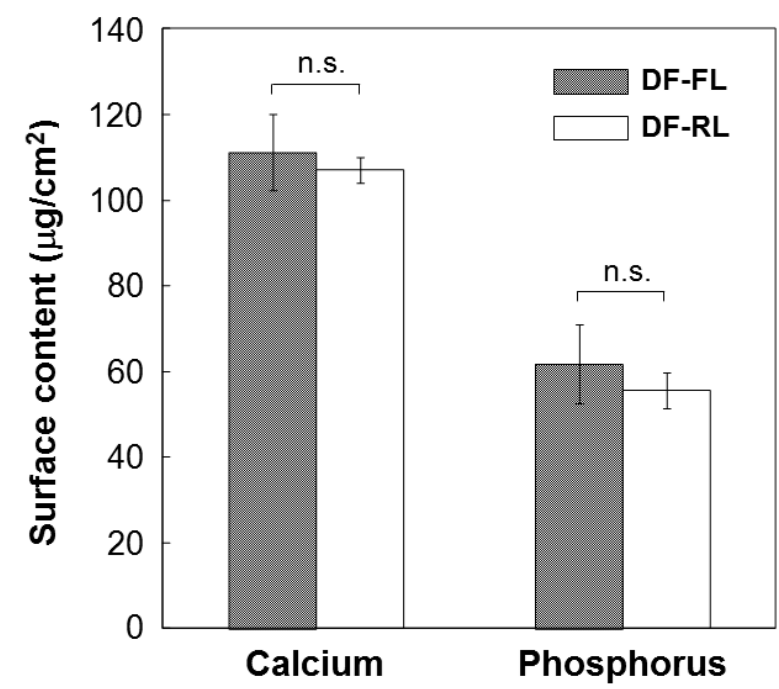

(a)

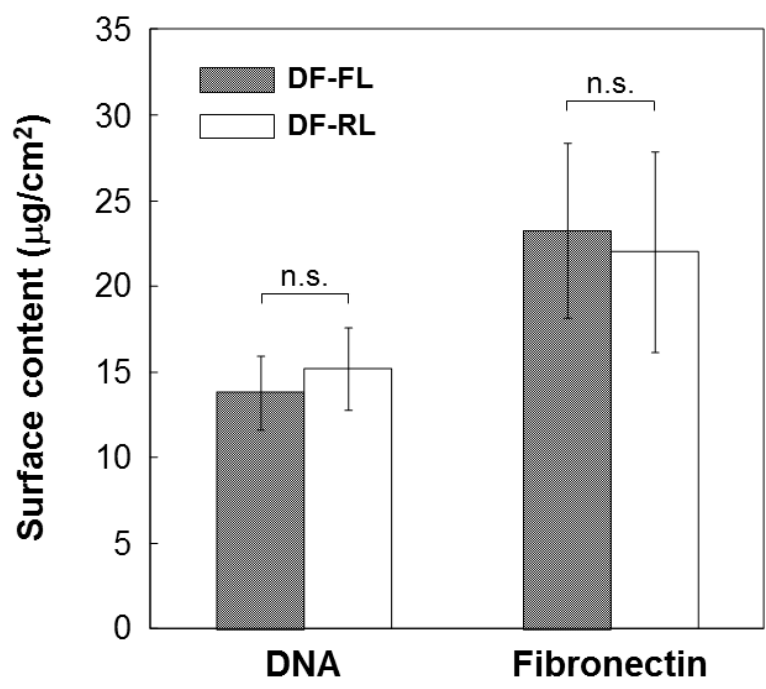

(b)

Figure 4. Contents of (a) calcium and phosphorus; and (b) DNA and fibronectin of the DNA-fibronectin-apatite composite layers (DF-Ap layers) immobilizing firefly luciferase $(F L)$ gene (Sample DF-FL) and Renilla luciferase (RL) gene (Sample DF-RL). The results shown are the mean and standard deviation of three independent experiments.

\subsection{Cotransfer of Reporter Genes by Conventional Lipofection}

To evaluate the trans effect of the promoters in the pGL-3 and pRL-TK vectors, a preliminary cotransfer study was performed using the conventional particle-mediated lipofection system. The mass percentage composition of pGL-3:pRL-TK was set at either 50:50 or 5:95, with a retention of a constant total DNA dose. When the percentage composition of pGL3:pRL-TK was 50:50 (in mass \%), the cotransfected CHO-K1 cells exhibited the RL activity $(4.6 \times 10$ and $3.3 \times 10$ counts in two independent experiments) that was four orders of magnitude lower compared with the FL activity $\left(6.3 \times 10^{5}\right.$ and 
$5.8 \times 10^{5}$ counts). When the percentage composition of pGL3:pRL-TK was changed from 50:50 to 5:95, the RL activity $\left(4.5 \times 10^{4}\right.$ and $1.6 \times 10^{4}$ counts $)$ of the cells increased by three orders of magnitude and got closer to the FL activity $\left(1.6 \times 10^{5}\right.$ and $2.5 \times 10^{5}$ counts). At this percentage composition (pGL3:pRL-TK = 5:95), the dose of pRL-TK was approximately twenty times as much as that of pGL3. Despite this, the RL activity of the cells was still slightly lower than the FL activity.

\subsection{Dual Reporter Gene Transfer Using a Pair of DF-Ap Layers}

Area-specific dual gene transfer on the DF-Ap layers was successfully demonstrated using the $F L$ and $R L$ reporter genes. As shown in Figure 5, the FL activity of the CHO-K1 cells cultured on Sample DF-FL was approximately two orders of magnitude higher than that of the cells cultured on Sample DF-RL. On the other hand, the RL activity of the cells cultured on Sample DF-RL was approximately three orders of magnitude higher than that of the cells cultured on Sample DF-FL. The FL and RL activities of the cells cultured on Samples DF-RL and DF-FL, respectively, were both at the background level, i.e., the luminescence level in the measurement without any test solution.

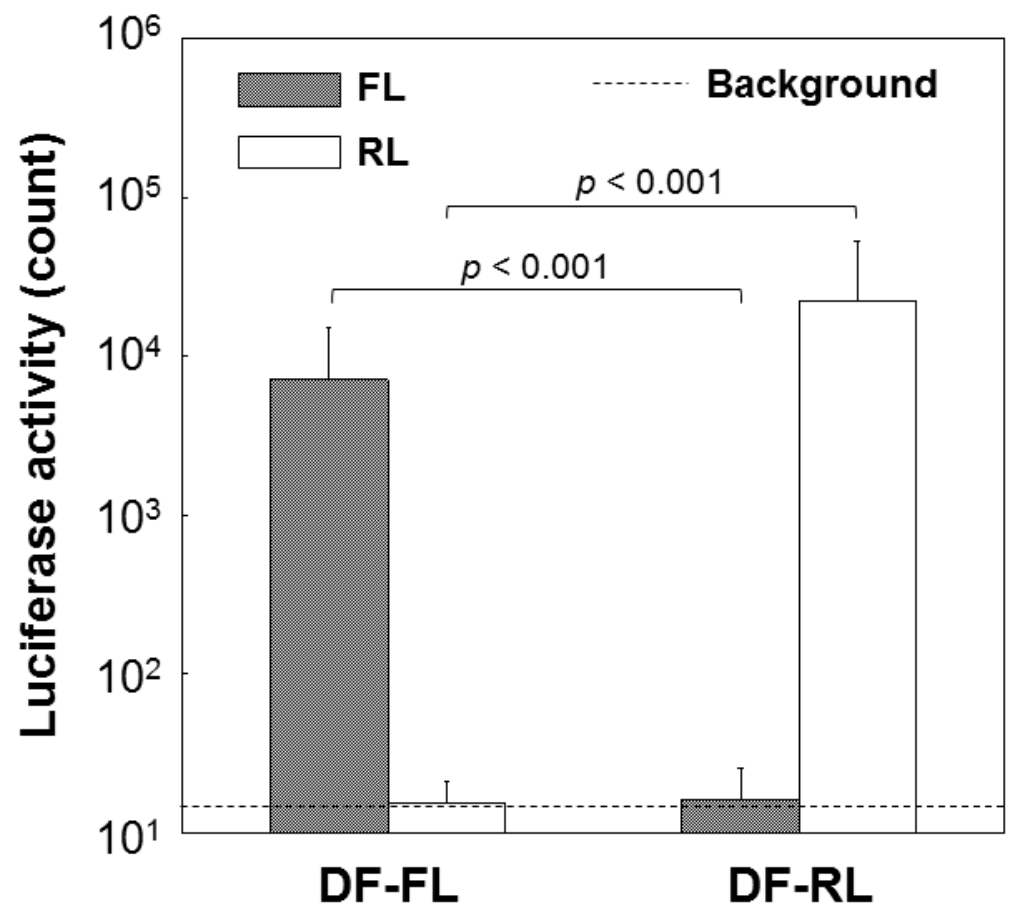

Figure 5. Luciferase (firefly luciferase (FL) and Renilla luciferase (RL)) activity in CHO-K1 cells cultured on the DNA-fibronectin-apatite composite layers (DF-Ap layers) immobilizing FL gene (Sample FL) and $R L$ gene (Sample DF-RL). Both Samples FL and RL were adjacently placed in one well of a four-well chamber slide. The results shown are the mean and standard deviation of five independent experiments.

Note that preparation conditions of the DF-Ap layers (to be described in Section 4.3) were decided to maximize the fibronectin content and gene transfer efficiency of the layer [21]. As shown in Figure 6, the CHO-K1 cells adhered well to the surface of the DF-Ap layer, most likely due to the cell adhesion activity of fibronectin immobilized in the DF-Ap layer [21]. 


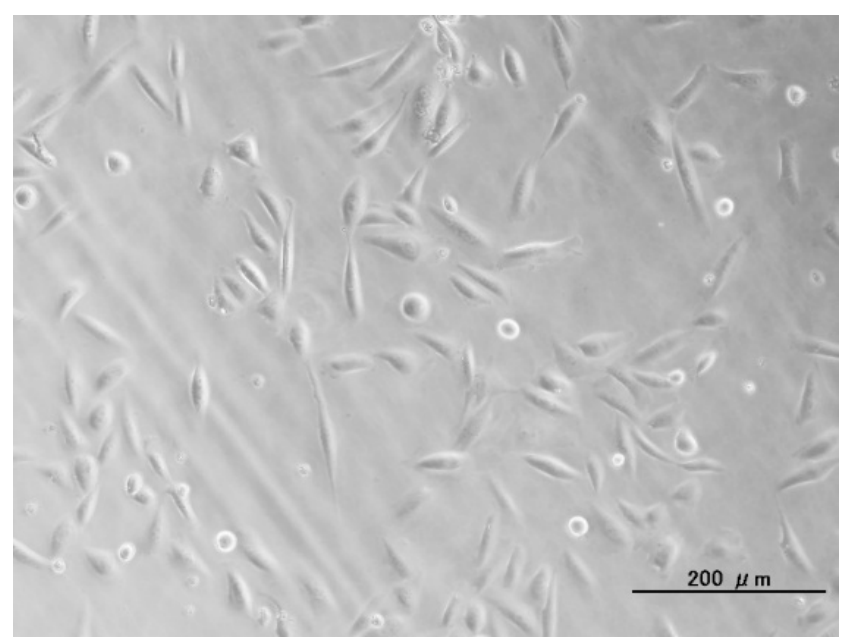

Figure 6. Optical microscopy image of CHO-K1 cells cultured on the DNA-fibronectin-apatite composite layer (DF-Ap layer) immobilizing firefly luciferase (FL) gene (Sample DF-FL). The scale bar indicates $200 \mu \mathrm{m}$.

\subsection{Dual Differentiation Factor Gene Transfer Using a Pair of DF-Ap Layers}

We performed preliminary dual gene transfer studies using the differentiation factor genes $V E G F$ and $B M P-2$ and multipotential C3H10T1/2 cells. Generally, gene transfer to multipotential cell lines like $\mathrm{C} 3 \mathrm{H} 10 \mathrm{~T} 1 / 2$ is more difficult than that to easy-to-transfect cell lines like CHO-K1. Despite this, our gene transfer system using the DF-Ap layer was considered to be valid also for the C3H10T1/2 cells. Preliminary real-time PCR results of two independent experiments indicated a potential increase (2-4 orders of magnitudes) in VEGF expression level in the C3H10T1/2 cells cultured on Sample DF-V compared with that in the cells cultured on Samples DF-B and F (negative control) with a fibronectin-apatite composite layer. The real-time PCR results also suggested 2-4 orders of magnitudes higher BMP-2 expression level in the C3H10T1/2 cells cultured on Sample DF-B compared with that in the cells cultured on Samples DF-V and F.

The real-time PCR results showed a sign of different expression patterns of differentiation marker genes in the C3H10T1/2 cells depending on the type of samples used for cell culturing: Samples F, DF-V, and DF-B. The results indicated a potential increase (3.3-fold in one experiment and 3.7-fold in the other experiment) in expression level of the endothelial differentiation marker CD31 in the cells cultured on Sample DF-V than that in the cells cultured on Sample F. The cells cultured on Sample DF-B showed no sign of enhanced CD31 expression level compared with the cells cultured on Sample F. On the other hand, the cells cultured on Sample DF-B showed a sign of enhanced expression level of osteogenic differentiation marker alkaline phosphatase (ALP) (3.4-fold in one experiment and 5.6-fold in the other experiment) compared with the cells cultured on Sample F. These preliminary results using the differentiation factor genes support the results of dual gene transfer studies using the $F L$ and $R L$ reporter genes (Section 2.3).

\section{Discussion}

Four kinds of DF-Ap layers were fabricated using the $F L$ and $R L$ reporter genes and the $V E G F$ and BMP-2 differentiation factor genes (Table 1). According to SEM (Figure 2), TF-XRD (Figure 3), 
ICP (Figure 4a), and UV-Vis (Figure 4b) results, these layers were similar in their surface morphology, crystalline phase, and the contents of apatite, plasmid DNA, and fibronectin, all of which are controlling factors that affect gene transfer efficiency $[21,25,46]$. The critical difference among these DF-Ap layers lies only on the type of plasmid DNA that was immobilized within the layer. Therefore, the effect of the physiochemical factors of the DF-Ap layers on gene expression efficiency was possibly negligible in the subsequent gene transfer studies.

The dual gene transfer studies indicated that the DF-Ap layers have the potential to mediate area-specific dual gene transfer and to stimulate cells on an area-by-area basis, even in the same well. As shown in Figure 5, the CHO-K1 cells cultured on the pGL3-derived DF-Ap layer showed only FL activity. In contrast, the cells cultured on the pRL-TK-derived DF-Ap layer exhibited only RL activity. The cotransfer of the $F L$ and $R L$ genes into the cells was unlikely to occur, taking into account the performance of the pGL3 and pRL-TK vectors, which was compared by the preliminary cotransfer study (Section 2.2). In the preliminary cotransfer study using conventional lipofection, the cotransfected CHO-K1 cells exhibited a slightly higher FL activity than RL activity, even when the dose of pGL3 was only approximately one twentieth that of pRL-TK (pGL3:pRL-TK = 5:95). This may be attributed to the trans effect [47] between the promoters/enhancers present in pGL3 and pRL-TK, i.e., the promoters and enhancers in pGL3, which included the $F L$ gene, were stronger than those in pRL-TK, which included the $R L$ gene. Therefore, even if only a small amount (e.g., one twentieth of $R L$ ) of the $F L$ gene from the pGL3-derived DF-Ap layer (on Sample DF-FL) was cotransferred into the cells on the pRL-TK-derived DF-Ap layer (on Sample DF-RL), those cells should have exhibited not only $R L$ but also $F L$ gene expression. Experimental results showed that this is not the case; the cells on the pRL-TK-derived DF-Ap layer exhibited only RL activity (Figure 5). From the results described above, cross-transfection from the two neighboring DF-Ap layers is likely to be denied. It is suggested that the plasmid DNA immobilized in the DF-Ap layer is locally transferred to the cells that adhered to the layer and hardly to the neighboring cells that were not in contact with this layer, as reported in our preliminary study [21].

The area-specific dual gene transfer on the DF-Ap layers was supported by the preliminary dual differentiation factor gene transfer study. As described in Section 2.4., the C3H10T1/2 cells cultured on Samples DF-V and DF-B were likely to proliferate into the cells with different gene expression patterns depending on the differentiation factor genes (VEGF and BMP-2) that were immobilized in the DF-Ap layer, even in the same well. The preliminary real-time PCR results suggested the enhanced $C D 31$ expression level in the $\mathrm{C} 3 \mathrm{H} 10 \mathrm{~T} 1 / 2$ cells on the DF-Ap layer immobilizing VEGF gene (Sample DF-V) than in the cells on the fibronectin-apatite composite layer (Sample F). The results also suggested the enhanced $A L P$ expression level in the cells on the DF-Ap layer immobilizing BMP-2 gene (Sample DF-B) than in the cells on the fibronectin-apatite composite layer (Sample F). The expression of these differentiation markers $(C D 31, A L P)$ in the cells may be stimulated because of the enhanced expression of the differentiation factor genes (VEGF, $B M P-2)$. Note that there seemed to be no noticeable difference in $C D 31$ expression level between the cells cultured on the DF-Ap layer immobilizing BMP-2 gene (Sample DF-B) and the cells cultured on the fibronectin-apatite composite layer (Sample F). This could be caused by the area-specific dual gene transfer on the DF-Ap layers, as demonstrated in Figure 5. Although more detailed analyses are required in the future, these preliminary results suggest that, as a 
consequence of dual gene transfer, the $\mathrm{C} 3 \mathrm{H} 10 \mathrm{~T} 1 / 2$ cells may have the potential to change into a different type of cells on Samples DF-V and Sample DF-B.

Considering the efficiency of our gene transfer system, approximately $7 \mu \mathrm{g}$ of plasmid DNA was immobilized within the DF-Ap layer on a single substrate $(10 \mathrm{~mm} \times 5 \mathrm{~mm})$ (Figure $4 \mathrm{~b})$ and transferred to the cells, resulting in approximately $10^{4}$ counts in the luciferase assay (Figure 5). In the conventional particle-mediated lipofection system, $0.4 \mu \mathrm{g}$ of plasmid DNA was used, resulting in approximately $10^{6}$ counts in the luciferase assay (data not shown). These findings suggest that the efficiency of our gene transfer system using the DF-Ap layer is not as high as the conventional lipofection system. However, our gene transfer system is valid in the presence of serum, provides a biocompatible surface [18] with good cell adhesion property (Figure 6), allows slow and sustained release of DNA [20,22,27], and is capable of stimulating cells on an area-by-area basis as described in the following paragraph, which would be advantages in tissue engineering applications.

In the present study, the cells were cultured on a pair of DF-Ap layers together in the same medium in the same well, and these layers were placed next to each other at a very narrow distance (approximately $100 \mu \mathrm{m}$ ) (Figure 1). In such situations, area-specific dual gene transfer could be difficult when using conventional particle-mediated systems because of the diffusion of DNA complexes in aqueous media. Therefore, the surface-mediated system using the DF-Ap layers is advantageous over conventional particle-mediated systems in terms of the spatial control of gene transfer. According to our previous results, area-specific gene transfer is possible not only with the DF-Ap layers but also with other CaP-based composite layers [26]. Moreover, CaP-based composite layers can be fabricated on various substrate materials using the precursor-assisted biomimetic process [25]. On the other hand, CaP-based composite layers can be fabricated on an intended area/position of substrate materials using area-specific CaP coating techniques (e.g., laser-assisted biomimetic process $[48,49]$ ). In combination with such an area-specific coating technique, multiple CaP-based composite layers that immobilize different genes could be patterned on a scaffold surface. Using an appropriate design of scaffolds and choice of genes for immobilization, it may be possible to produce the intended cell type at an intended area/position on a scaffold, and eventually, highly structured tissues that comprise different types of cells. However, this is a challenge that will be addressed in a future study.

\section{Materials and Methods}

\subsection{Materials}

Four types of plasmid DNAs $(0.7-1.2 \mathrm{mg} / \mathrm{mL})$ were prepared from four different genes (Table 1). A pGL3-Control vector including the cDNA of FL (Promega Corporation, Fitchburg, WI, USA) and a pRL-TK vector including the cDNA of $R L$ (Promega) were used in the reporter gene transfer studies. The pGL3-Control vector contains the SV40 promoter/enhancer, whereas the pRL-TK vector includes the herpes simplex virus (HSV) promoter. The pCI-neo vector (Promega) with the human cytomegalovirus (CMV) enhancer/promoter was employed in the cell differentiation study using the VEGF and BMP-2 genes. The cDNA of human $V E G F$ and that of human $B M P-2$ were inserted into the multiple cloning site of pCI-neo using the SalI and NotI sites at the linker ends (the resulting plasmid DNAs are termed pCI- $V E G F$ and pCI-BMP, respectively). The cDNAs of $V E G F$ and $B M P-2$ were cloned from HeLa cells 
by reverse transcription PCR. The cDNA of $V E G F$ was amplified using the following primers: forward primer, 5'-AGAGTCGACCTACCTCCACCATGCCAAGT-3' and reverse primer, 5'-ACTGCGGCCG CTGGTGAGAGATCTGGTTCCC-3'. The cDNA of BMP-2 was amplified using the following primers: forward primer, 5'-AGAGTCGACTGAGCCTTTCCAGCAAGTTT-3' and reverse primer, 5'-ACTGCG GCCGCGGAACGTGTGTGTGTGGTGT-3'.

Polystyrene plates with 1-mm thickness were prepared by hot pressing polystyrene pellets (Sigma-Aldrich, St. Louis, MO, USA). The polystyrene plates were cut into $10 \mathrm{~mm} \times 5 \mathrm{~mm}$ rectangular substrates, polished on both sides with a polishing compound (Tamiya polishing compound (Finish), Tamiya, Japan), washed ultrasonically with ethanol, and dried in a vacuum at $100{ }^{\circ} \mathrm{C}$ for $24 \mathrm{~h}$. Fibronectin $(1 \mathrm{mg} / \mathrm{mL})$ from bovine plasma was purchased from Sigma-Aldrich, USA. Chemical reagents other than those listed above for the DF-Ap layer preparation were purchased from Nacalai Tesque Inc., Kyoto, Japan.

The CHO-K1 ovary cells (RIKEN BioResource Center, Tsukuba, Japan) and C3H10T1/2 embryonic cells (RIKEN BioResource Center) were used in the dual reporter gene and differentiation factor gene transfer studies, respectively (Table 1). RPMI1640 (Life Technologies Corporation, Carlsbad, CA, USA) and BME medium (Life Technologies Corporation) were used as the culture media for the CHO-K1 and C3H10T1/2 cells, respectively. Both culture media were supplemented with fetal bovine serum (Life Technologies Corporation) at a concentration of $10 \%$.

\subsection{Alternate Dipping Treatment for Precoating with the Apatite Precursor}

The polystyrene substrate was subjected to oxygen plasma and subsequently to alternate dipping treatments to precoat with amorphous calcium phosphate (ACP), which is an apatite precursor [50]. The plasma treatment was performed in $\mathrm{O}_{2}$ gas using a compact ion etcher (FA-1, SAMCO Inc., Kyoto, Japan) at a pressure of $30 \mathrm{~Pa}$ and an energy density of $0.5 \mathrm{~W} / \mathrm{cm}^{2}$ under an electric field operating at 13.56 $\mathrm{MHz}$ for $30 \mathrm{~s}$ [50]. The plasma-treated polystyrene substrate was alternately dipped in calcium and phosphate ion solutions, as described elsewhere [50,51]. In brief, the substrate was dipped in $20 \mathrm{~mL}$ of a 50:50 (in vol \%) mixture of ethanol and $200 \mathrm{mM} \mathrm{CaCl}_{2}$ for $10 \mathrm{~s}$, then dipped in $20 \mathrm{~mL}$ of 50 vol \% aqueous ethanol for $1 \mathrm{~s}$, and dried in air for a few minutes. The substrate was subsequently dipped in $20 \mathrm{~mL}$ of a 50:50 (in vol \%) mixture of ethanol and $200 \mathrm{mM} \mathrm{K} 2 \mathrm{HPO}_{4} \cdot 3 \mathrm{H}_{2} \mathrm{O}$ for $10 \mathrm{~s}$, dipped again in $20 \mathrm{~mL}$ of $50 \mathrm{vol} \%$ aqueous ethanol for $1 \mathrm{~s}$, and dried in air for a few minutes. These alternate dipping operations in calcium and phosphate ion solutions were performed three times. The ACP-precoated polystyrene substrate was dried and then sterilized with ethylene oxide gas.

\subsection{Biomimetic Process for Coating with DF-Ap Layers}

Four kinds of coating solutions containing different plasmid DNAs (pGL-3, pRL-TK, pCI-VEGF, or pCI-BMP) and one control coating solution without plasmid DNA were prepared. First, a supersaturated $\mathrm{CaP}$ solution $\left(142 \mathrm{mM} \mathrm{NaCl}, 1.50 \mathrm{mM} \mathrm{K} 2 \mathrm{HPO}_{4} \cdot 3 \mathrm{H}_{2} \mathrm{O}, 3.75 \mathrm{mM} \mathrm{CaCl}, 50 \mathrm{mM}\right.$ tris(hydroxymethyl)aminomethane (Tris) [52]) was prepared by dissolving $\mathrm{NaCl}, \mathrm{K}_{2} \mathrm{HPO}_{4} \cdot 3 \mathrm{H}_{2} \mathrm{O}$, $\mathrm{HCl}(40 \mathrm{mM})$, and $\mathrm{CaCl}_{2}$ in ultrapure water and then buffering the solution at $\mathrm{pH}=7.40$ at $25.0{ }^{\circ} \mathrm{C}$ with Tris and $\mathrm{HCl}$ [19-22,52]. Coating solutions were prepared by adding each plasmid DNA and fibronectin at a concentration of 40 and $10 \mu \mathrm{g} / \mathrm{mL}$, respectively, to the CaP solution. The control 
coating solution was prepared by adding only fibronectin at a concentration of $10 \mu \mathrm{g} / \mathrm{mL}$ to the $\mathrm{CaP}$ solution. The plasmid DNA and fibronectin concentrations were determined according to our previous optimization results [21]. The coating solutions were sterilized by filtration.

The ACP-precoated polystyrene substrate (obtained in Section 4.2) was aseptically immersed in $1.5 \mathrm{~mL}$ of each coating solution at $25{ }^{\circ} \mathrm{C}$ for $24 \mathrm{~h}$. After removal from the coating solution, the substrate was gently washed with ultrapure water before surface analysis (Section 4.4), or washed with phosphate-buffered saline (PBS) before cell culturing (Sections 4.6-4.8). The samples that were prepared using pGL3, pRL-TK, pCI-VEGF, and pCI-BMP plasmid DNAs were denoted as Samples DF-FL, DF-RL, DF-V, and DF-B, respectively (Table 1). The sample that was prepared using the control coating solution without plasmid DNA was denoted as Sample F. Sample F had a fibronectin-apatite composite layer on its surface [21], which was used as a negative control in the dual differentiation factor gene transfer study (Section 4.8).

\subsection{Analysis of Sample Surfaces and Coating Solutions}

The surface morphologies and structures of Samples DF-FL and DF-RL were examined by SEM (XL30, FEI Company Ltd., Hillsboro, OR, USA) and TF-XRD (RINT Ultima X, Rigaku Co., Tokyo, Japan) employing CuK $\alpha$ X-rays.

The coating solutions were clear and induced no spontaneous precipitation during the biomimetic process ( $24 \mathrm{~h}$ immersion of the substrate). Changes in the calcium and phosphorus concentrations and plasmid DNA and fibronectin concentrations of the coating solutions during the biomimetic process were quantified by ICP (Model PS 7800, Hitachi High-Tech Science Co., Tokyo, Japan) and UV-Vis (Model UV-2450; Shimadzu Corporation, Kyoto, Japan), respectively. To determine plasmid DNA concentration, the absorbance was measured at $260 \mathrm{~nm}$. To assess fibronectin concentration, the absorbance was measured at $595 \mathrm{~nm}$ using a protein assay kit (Bio-Rad Laboratories, Inc., Hercules, CA, USA) based on the Bradford method. The content of calcium, phosphorus, plasmid DNA, and fibronectin immobilized on the sample surfaces was estimated by subtracting their final concentrations from the initial concentrations in the coating solution. Three substrates were used for each type of sample to obtain a mean value and a standard deviation. The data were compared using Student's $t$-test, where the significance level was set at $p<0.05$ for each analysis.

\subsection{Cotransfer of Reporter Genes by Conventional Lipofection}

Cotransfection was conducted using the commercial lipid transfection reagent Lipofectamine (Life Technologies Corporation), in accordance with the manufacturer's recommended optimum conditions. First, the CHO-K1 cells were seeded in each well of a 24 -well cell culture plate at a cell concentration of $5 \times 10^{4}$ cells $/ 0.5 \mathrm{~mL} /$ well. After culturing for $24 \mathrm{~h}$ in RPMI1640 medium with serum, the medium was replaced with $0.2 \mathrm{~mL}$ of serum-free Opti-MEM (Life Technologies Corporation) and then supplemented with $0.2 \mathrm{~mL}$ of serum-free Opti-MEM containing $0.4 \mu \mathrm{g}$ of plasmid DNA (pGL-3 and pRL-TK) and $4 \mu \mathrm{L}$ of the lipid transfection reagent. The mass percentage composition of pGL-3:pRL-TK was set at either 50:50 or 5:95, with a retention of a constant total DNA dose. After culturing for $5 \mathrm{~h}$, the medium was replaced again with a serum-containing RPMI1640 medium. After culturing for another $43 \mathrm{~h}$, the cells were washed three times with PBS and lysed in $200 \mu \mathrm{L}$ of Passive 
Lysis Buffer (Promega). After vortexing, the cell lysate was centrifuged at 13,370× $g$ for 2 min. The supernatant was analyzed using a dual luciferase assay kit (Promega) and a luminometer (Gene Light 55, Micro-tec Co., Ltd., Chiba, Japan) to evaluate the FL and RL activities of the cells.

\subsection{Observation of Cells on DF-Ap Layer}

Sample DF-FL was placed in one well of a twenty-four-well culture plate. The CHO-K1 cells were seeded on the sample at a cell concentration of $2.5 \times 10^{4}$ cells $/ 0.5 \mathrm{~mL} /$ well. After culturing for $48 \mathrm{~h}$, the cells on the sample surface were observed by transmission optical microscopy (IX71, Olympus Co., Tokyo, Japan).

\subsection{Dual Reporter Gene Transfer Using a Pair of DF-Ap Layers}

As shown in Figure 1, Samples DF-FL and DF-RL were adjacently placed in one well of a four-well chamber slide (Nunc ${ }^{\mathrm{TM}}$ Lab-Tek $^{\mathrm{TM}}$ II Chamber Slide ${ }^{\mathrm{TM}}$ System). The CHO-K1 cells were seeded on the samples at a cell concentration of $5 \times 10^{4}$ cells $/ 0.5 \mathrm{~mL} /$ well. After culturing for $72 \mathrm{~h}$, the cells on each sample were assayed for FL and RL activities using the method described in the preceding section. Five substrates were used for each type of sample to obtain a mean value and a standard deviation. The data were compared using Student's $t$-test.

\subsection{Dual Differentiation Factor Gene Transfer Using a Pair of DF-Ap Layers}

Samples DF-V and DF-B were adjacently placed in one well of the four-well chamber slide in the same manner as that illustrated in Figure 1. Sample F was placed in the well of the four-well chamber slide alone as a negative control. The $\mathrm{C} 3 \mathrm{H} 10 \mathrm{~T} 1 / 2$ cells were seeded on the samples at a cell concentration of $5 \times 10^{3}$ cells $/ 0.5 \mathrm{~mL} /$ well and cultured for 14 days (the spent medium was replaced at 7 days of culture). After 14 days of culture, VEGF, BMP-2, CD31, and ALP gene expression was evaluated by real-time PCR (Mini Opticon real-time PCR system, Bio-Rad Laboratories Inc.). The cells cultured on each sample were washed three times with PBS, lysed, and centrifuged to obtain the supernatant. Total RNA was extracted from the supernatant using an RNA extraction kit (QIAGEN, Hilden, Germany). Five microgram of total RNA was reverse transcribed in a buffer containing $1 \mu \mathrm{L}$ of oligo-dT primers $(50 \mu \mathrm{M}), 1 \mu \mathrm{L}$ of deoxynucleotides (10 mM each), $20 \mathrm{U}$ of RNase inhibitor (Takara Bio Inc., Shiga, Japan), and $200 \mathrm{U}$ of PrimeScript ${ }^{\circledR}$ RTase (Takara Bio). This mixture was incubated for $45 \mathrm{~min}$ at $42^{\circ} \mathrm{C}$ and for $5 \mathrm{~min}$ at $95^{\circ} \mathrm{C}$. VEGF, BMP-2,CD31, ALP, and $\beta$-actin gene expression levels were detected using the following primers: forward primer 5'-AAGGAGGAGGGCAGAATCAT-3' and reverse primer 5'-ATGTTGGACTCCTCAGTGGG-3' for human VEGF; forward primer 5'-AAGGCA CCCTTTGTATGTGG-3' and reverse primer 5'-CATGCCTTAGGGATTTTGGA-3' for human BMP-2; forward primer 5'-GCCCAATCACGTTTCAGTTT-3' and reverse primer 5'-GGCTTCCACACTAGGC TCAG-3' for mouse CD31; forward primer 5'-GAGCAGGAACACAAGTTTGC-3' and reverse primer 5'-GTTGCAGGGTCTGGAGAGTA-3' for mouse $A L P$; and forward primer 5'-GGACCTGGCTGGCC GGGACC-3' and reverse primer 5'-GCGGTGCACGATGGAGGGGC-3' for mouse $\beta$-actin. Each primer $(12.5 \mathrm{pM}$ ) was added to a test solution containing $12.5 \mu \mathrm{L}$ of iQ SYBR green supermix (Bio-Rad Laboratories) together with $0.5 \mu \mathrm{L}$ of template sample (final volume, $25 \mu \mathrm{L}$ ). The gene expression levels 
were expressed as the delta-delta cycle time $[\Delta-\Delta C(\mathrm{t})]$, which was normalized to the $\beta$-actin expression level. Two substrates were used for each type of sample in the real-time PCR assay.

\section{Conclusions}

We successfully demonstrated that the DF-Ap layers can mediate area-specific dual gene transfer. The cells cultured on two different DF-Ap layers exhibited different gene expression patterns depending on the gene that was immobilized in the underlying layer, even in the same well. DF-Ap layers with area-specific cell stimulation capability could be useful in tissue engineering applications.

\section{Acknowledgments}

This work was supported by KAKENHI (Grants-in-Aid for Scientific Research) Grant Number 22700499, 26560250 from Japan Society for the Promotion of Science (JSPS). A part of this work was conducted at the Nano-Processing Facility, supported by IBEC Innovation Platform, National Institute of Advanced Industrial Science and Technology (AIST), Japan.

\section{Author Contributions}

Ayako Oyane and Hideo Tsurushima conceived and designed the experiments; Yushin Yazaki performed the experiments; Atsuo Ito, Yu Sogo, and Atsushi Yamazaki helped with the evaluation of the experiments; Yushin Yazaki and Ayako Oyane wrote the paper.

\section{Conflicts of Interest}

The authors declare no conflict of interest.

\section{References}

1. Bennett, M.J.; Aberle, A.M.; Balasubramaniam, R.P.; Malone, J.G.; Malone, R.W.; Nantz, M.H. Cationic lipid-mediated gene delivery to murine lung: Correlation of lipid hydration with in vivo transfection activity. J. Med. Chem. 1997, 40, 4069-4078.

2. Templeton, N.S.; Lasic, D.D.; Frederik, P.M.; Strey, H.H.; Roberts, D.D.; Pavlakis, G.N. Improved DNA: Liposome complexes for increased systemic delivery and gene expression. Nat. Biotechnol. 1997, 15, 647-652.

3. Uduehi, A.N.; Moss, S.H.; Nuttall, J.; Pouton, C.W. Cationic lipid-mediated transfection of differentiated Caco-2 cells: A filter culture model of gene delivery to a polarized epithelium. Pharm. Res. 1999, 16, 1805-1811.

4. Abdallah, B.; Sachs, L.; Demeneix, B.A. Non-viral gene transfer: Applications in developmental biology and gene therapy. Biol. Cell 1995, 85, 1-7.

5. Boussif, O.; Lezoualc'h, F.; Zanta, M.A.; Mergny, M.D.; Scherman, D.; Demeneix, B.; Behr, J.P. A versatile vector for gene and oligonucleotide transfer into cells in culture and in vivo: Polyethylenimine. Proc. Natl. Acad. Sci. USA 1995, 92, 7297-7301. 
6. Felgner, P.L.; Barenholz, Y.; Behr, J.P.; Cheng, S.H.; Cullis, P.; Huang, L.; Jessee, J.A.; Seymour, L.; Szoka, F.; Thierry, A.R.; et al. Nomenclature for synthetic gene delivery systems. Hum. Gene Ther. 1997, 8, 511-512.

7. Gebhart, C.L.; Kabanov, A.V. Evaluation of polyplexes as gene transfer agents. J. Control. Release 2001, 73, 401-416.

8. Graham, F.L.; van der Eb A.J. A new technique for the assay of infectivity of human adenovirus 5 DNA. Virology 1973, 52, 456-467.

9. Batard, P.; Jordan, M.; Wurm, F. Transfer of high copy number plasmid into mammalian cells by calcium phosphate transfection. Gene 2001, 270, 61-68.

10. Roy, I.; Mitra S.; Maitra, A.; Mozumdar, S. Calcium phosphate nanoparticles as novel non-viral vectors for targeted gene delivery. Int. J. Pharm. 2003, 250, 25-33.

11. Chowdhury, E.H.; Kunou, M.; Harada, I.; Kundu, A.K.; Akaike, T. Dramatic effect of $\mathrm{Mg}^{2+}$ on transfecting mammalian cells by DNA/calcium phosphate precipitates. Anal. Biochem. 2004, 328, 96-97.

12. Kakizawa, Y.; Furukawa, S.; Kataoka, K. Block copolymer-coated calcium phosphate nanoparticles sensing intracellular environment for oligodeoxynucleotide and siRNA delivery. J. Control. Release 2004, 97, 345-356.

13. Kutsuzawa, K.; Chowdhury, E.H.; Nagaoka, M.; Maruyama, K.; Akiyama, Y.; Akaike, T. Surface functionalization of inorganic nano-crystals with fibronectin and E-cadherin chimera synergistically accelerates trans-gene delivery into embryonic stem cells. Biochem. Biophys. Res. Commun. 2006, 350, 514-520.

14. Shea, L.D.; Smiley, E.; Bonadio, J.; Mooney, D.J. DNA delivery from polymer matrices for tissue engineering. Nat. Biotechnol. 1999, 17, 551-554.

15. Ziauddin, J.; Sabatini, D.M. Microarrays of cells expressing defined cDNAs. Nature 2001, 411, 107-110.

16. Ono, I.; Yamashita, T.; Jin, H.Y.; Ito, Y.; Hamada, H.; Akasaka, Y.; Nakasu, M.; Ogawa, T.; Jimbow, K. Combination of porous hydroxyapatite and cationic lipids as a vector for BMP-2 gene therapy. Biomaterials 2004, 25, 4709-4718.

17. Yoshikawa, T.; Uchimura, E.; Kishi, M.; Funeriu, D.P.; Miyake, M.; Miyake, J. Transfection microarray of human mesenchymal stem cells and on-chip siRNA gene knockdown. J. Control. Release 2004, 96, 227-232.

18. Shen, H.; Tan, J.; Saltzman, W.M. Surface-mediated gene transfer from nanocomposites of controlled texture. Nat. Mater. 2004, 3, 569-574.

19. Oyane, A.; Tsurushima, H.; Ito, A. Novel gene-transferring scaffolds having a cell adhesion molecule-DNA-apatite nanocomposite surface. Gene Ther. 2007, 14, 1750-1753.

20. Oyane, A.; Tsurushima, H.; Ito, A. Highly efficient gene transfer system using laminin-DNA-apatite composite layer. J. Gene Med. 2010, 12, 194-206.

21. Oyane, A.; Murayama, M.; Yamazaki, A.; Sogo, Y.; Ito, A.; Tsurushima, H. Fibronectin-DNA-apatite composite layer for highly efficient and area-specific gene transfer. J. Biomed. Mater. Res. A 2010, 92A, 1038-1047. 
22. Yazaki, Y.; Oyane, A.; Tsurushima, H.; Sogo, Y.; Ito, A.; Yamazaki, A. Control of gene transfer on a DNA-fibronectin-apatite composite layer by the incorporation of carbonate and fluoride ions. Biomaterials 2011, 32, 4896-4902.

23. Sun, B.B.; Tran, K.K.; Shen, H. Enabling customization of non-viral gene delivery systems for individual cell types by surface-induced mineralization. Biomaterials 2009, 30, 6386-6393.

24. Luong, L.N.; McFalls, K.M.; Kohn, D.H. Gene delivery via DNA incorporation within a biomimetic apatite coating. Biomaterials 2009, 30, 6996-7004.

25. Oyane, A.; Wang, X.P.; Sogo, Y.; Ito, A.; Tsurushima, H. Calcium phosphate composite layers for surface-mediated gene transfer. Acta Biomater. 2012, 8, 2034-2046.

26. Oyane, A.; Yazaki, Y.; Araki, H.; Sogo, Y.; Ito, A.; Yamazaki, A.; Tsurushima, H. Fabrication of a DNA-lipid-apatite composite layer for efficient and area-specific gene transfer. J. Mater. Sci. Mater. Med. 2012, 23, 1011-1019.

27. Yazaki, Y.; Oyane, A.; Tsurushima, H.; Araki, H.; Sogo, Y.; Ito, A.; Yamazaki, A. Coprecipitation of DNA-lipid complexes with apatite and comparison with superficial adsorption for gene transfer applications. J. Biomater. Appl. 2014, 28, 937-945.

28. He, C.X.; Tabata, Y.; Gao, J.Q. Non-viral gene delivery carrier and its three-dimensional transfection system. Int. J. Pharm. 2010, 386, 232-242.

29. Yamauchi, F.; Kato, K.; Iwata, H. Micropatterned, self-assembled monolayers for fabrication of transfected cell microarrays. Biochim. Biophys. Acta 2004, 1672, 138-147.

30. Chang, F.H.; Lee, C.H.; Chen, M.T.; Kuo, C.C.; Chiang, Y.L.; Hang, C.Y.; Roffler, S. Surfection: A new platform for transfected cell arrays. Nucleic Acids Res. 2004, 32, e33.

31. Baghdoyan, S.; Roupioz, Y.; Pitaval, A.; Castel, D.; Khomyakova, E.; Papine, A.; Soussaline, F.; Gidrol, X. Quantitative analysis of highly parallel transfection in cell microarrays. Nucleic Acids Res. 2004, 32, e77.

32. Jain, T.; Muthuswamy, J. Bio-chip for spatially controlled transfection of nucleic acid payloads into cells in a culture. Lab. Chip. 2007, 7, 1004-1011.

33. Yamauchi, F.; Kato, K.; Iwata, H. Layer-by-layer assembly of poly(ethyleneimine) and plasmid DNA onto transparent indium-tin oxide electrodes for temporally and spatially specific gene transfer. Langmuir 2005, 21, 8360-8367.

34. Yamauchi, F.; Kato, K.; Iwata, H. Spatially and temporally controlled gene transfer by electroporation into adherent cells on plasmid DNA-loaded electrodes. Nucleic Acids Res. 2004, 32, e187.

35. Inoue, Y.; Fujimoto, H.; Ogino, T.; Iwata, H. Site-specific gene transfer with high efficiency onto a carbon nanotube-loaded electrode. J. R. Soc. Interface 2008, 5, 909-918.

36. Nomikou, N.; McHale, A.P.; McHale, A.P. Exploiting ultrasound-mediated effects in delivering targeted; site-specific cancer therapy. Cancer Lett. 2010, 296, 133-143.

37. Thein, M.; Cheng, A.; Khanna, P.; Zhang, C.; Park, E.-J.; Ahmed, D.; Goodrich, C.J.; Asphahani, F.; Wu, F.; Smith, N.B.; et al. Site-specific sonoporation of human melanoma cells at the cellular level using high lateral-resolution ultrasonic micro-transducer arrays. Biosens. Bioelectron. 2011, 27, 25-33.

38. Suzuki, R.; Takizawa, T.; Negishi, Y.; Utoguchi, N.; Maruyama, K. Effective gene delivery with liposomal bubbles and ultrasound as novel non-viral system. J. Drug Target. 2007, 15, 531-537. 
39. Ogawa, Y.; Morikawa, N.; Ohkubo-Suzuki, A.; Miyoshi, S.; Arakawa, H.; Kita, Y.; Nishimura, S. An epoch-making application of discharge plasma phenomenon to gene-transfer. Biotechnol. Bioeng. 2005, 92, 865-870.

40. Sakai, Y.; Khajoee, V.; Ogawa, Y.; Kusuhara, K.; Katayama, Y.; Hara, T. A novel transfection method for mammalian cells using gas plasma. J. Biotechnol. 2006, 121, 299-308.

41. Connolly, R.J.; Lopez, G.A.; Hoff, A.M.; Jaroszeski, M.J. Plasma facilitated delivery of DNA to skin. Biotechnol. Bioeng. 2009, 104, 1034-1040.

42. Zhang, W.; Tsurushima, H.; Oyane, A.; Yazaki, Y.; Sogo, Y.; Ito, A.; Matsumura, A. BMP-2 gene-fibronectin-apatite composite layer enhances bone formation. J. Biomed. Sci. 2011, 18, 62.

43. Wang, X.; Ito, A.; Li, X.; Sogo, Y.; Hirose, M.; Oyane, A.; Tsurushima, H. DNA-lipid-apatite composite layers enhance gene expression of mesenchymal stem cells. Mater. Sci. Eng. C Mater. Biol. Appl. 2013, 33, 512-518.

44. Leung, D.W.; Cachianes, G.; Kuang, W.J.; Goeddel, D.V.; Ferrara, N. Vascular endothelial growth factor is a secreted angiogenic mitogen. Science 1989, 246, 1306-1309.

45. Wozney, J.M.; Rosen, V.; Celeste, A.J.; Mitsock, L.M.; Whitters, M.J.; Kriz, R.W.; Hewick, R.M.; Wang, E.A. Novel regulators of bone formation: Molecular clones and activities. Science 1988, $242,1528-1534$.

46. Oyane, A.; Araki, H.; Sogo, Y.; Ito, A.; Tsurushima, H. Spontaneous assembly of DNA-amorphous calcium phosphate nanocomposite spheres for surface-mediated gene transfer. CrystEngComm 2013, 15, 4994-4997.

47. Farr, A.; Roman, A. A pitfall of using a second plasmid to determine transfection efficiency. Nucleic Acids Res. 1992, 20, 920.

48. Lee, B.H.; Oyane, A.; Tsurushima, H.; Shimizu, Y.; Sasaki, T.; Koshizaki, N. A new approach for hydroxyapatite coating on polymeric materials using laser-induced precursor formation and subsequent aging. Appl. Mater. Interfaces 2009, 1, 1520-1524.

49. Oyane, A.; Sakamaki, I.; Shimizu, Y.; Kawaguchi, K.; Koshizaki, N. Liquid-phase laser process for simple and area-specific calcium phosphate coating. J. Biomed. Mater. Res. A 2012, 100A, 2573-2580.

50. Oyane, A.; Tsurushima, H.; Ito, A. Simple surface modification process to produce a transparent apatite-polystyrene composite for in situ observation of cell behavior. Chem. Lett. 2006, 35, 1300-1301.

51. Oyane, A.; Uchida, M.; Yokoyama, Y.; Choong, C.; Triffitt, J.; Ito, A. Simple surface modification of poly(e-caprolactone) to induce its apatite-forming ability. J. Biomed. Mater. Res. A 2005, 75A, $138-145$.

52. Uchida, M.; Oyane, A.; Kim, H.M.; Kokubo, T.; Ito, A. Biomimetic coating of laminin-apatite composite on titanium metal with excellent cell adhesive property. Adv. Mater. 2004, 16, 1071-1074.

(C) 2015 by the authors; licensee MDPI, Basel, Switzerland. This article is an open access article distributed under the terms and conditions of the Creative Commons Attribution license (http://creativecommons.org/licenses/by/4.0/). 